\title{
IRECIST Confirmed Progressive Disease
}

National Cancer Institute

\section{Source}

National Cancer Institute. iRECIST Confirmed Progressive Disease. NCI Thesaurus. Code C142356.

Radiographic progression which has been confirmed by additional imaging showing worsening of initial progression. 